III.

\title{
Ueber die feinere Struktur der hämoglobinlosen und hämoglobinhaltigen Knochenmarkzellen.
}

\author{
Von Prof. Dr. Julius Arnold in Heidelberg.
}

(Hierzu Taf. I und II.)

Die Ueberzeugung, dass das rothe Knochenmark für Biologen und Morphologen eines der ergiebigsten Objecte ist, hatte sich mir schon bei meinen ersten Untersuchungen (1882) aufgedrängt. Es gilt dies nicht nur bezüglich der Theilungsvorgänge, deren Erkenntniss durch die an den Knochenmarkzellen angestellteu Forschungen wesentlich bereichert und vertieft worden ist, sondern auch betreffs des feineren Baues des ruhenden Kerns und des Zellleibes. Es gehört keine prophetische Begabung zu der Voraussage, dass die Knochenmarkzellen in der Lehre von der sog. Protoplasmastruktur eine mindestens ebenso bedeutungsvolle Rolle spielen werden, wie in derjenigen von den Theilungsprozessen. - Bei der grossen Bedeutung, welche den Knochenmarkzellen wegen der an ihnen sich abspielenden Vermehrungsvorgänge, sowie ihrer sonstigen functionellen Leistungen für die Zusammensetzung des Blutes unter normalen und pathologischen Verhältnissen zukommt, ist dies gar nicht anders zu erwarten. Die nachfolgenden Mittheilungen beziehen sich ausschliesslich auf das rothe Knochenmark des Kaninchens und bitte ich diese als bescheidene Nachträge zu den früheren Beiträgen, den letzten insbesondere ${ }^{1}$ ), anzusehen. Vielleicht sind dieselben geeignet, auch Audere zur Bearbeitung des rothen Knochenmarkes anzuregen. Es wurden ja in den letzten Jahren durch verschiedene ebenso gründliche, als erfolgreiche Untersuchungen mehrere sehr bedeutungsvolle Thatsachen zu Tage gefördert. Ich bin aber der Zustimmung gerade dieser Autoren sicher, wenn ich behaupte,

1) J. Arnold, Zur Morphologie und Biologie der Zellen des Knochenmarkes. Dieses Archiv. Bd. 140. 1895. 

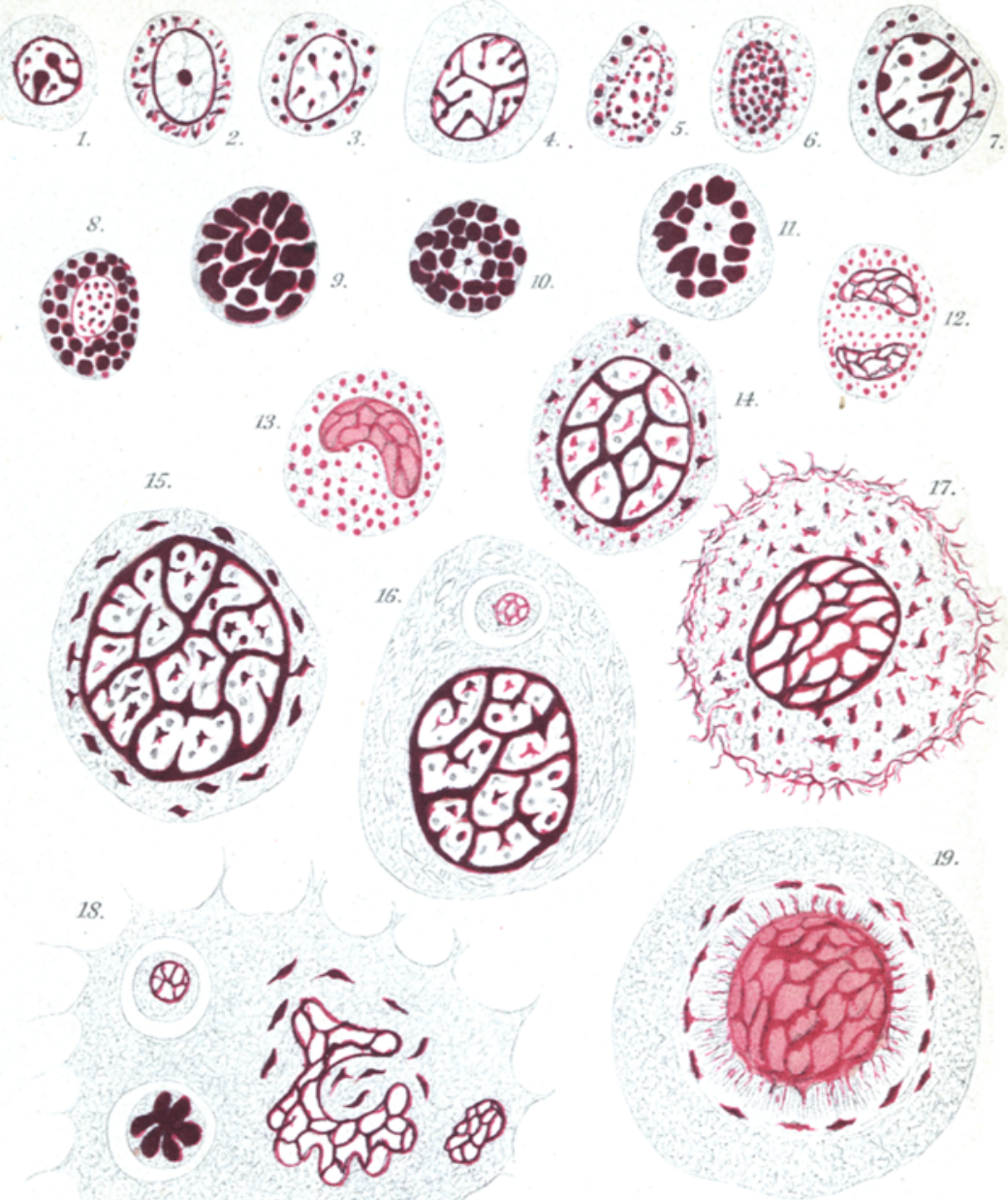

21.
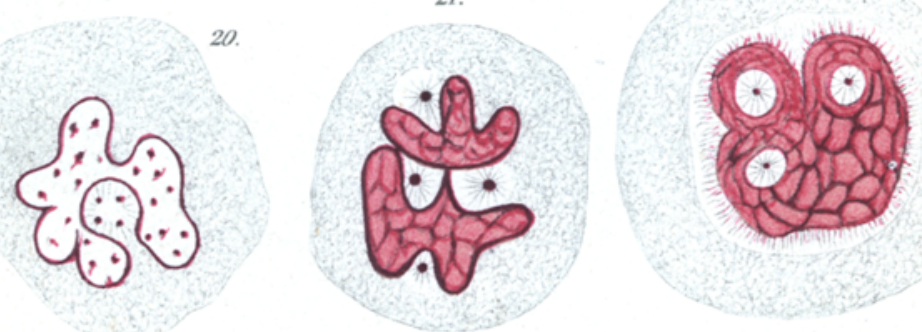

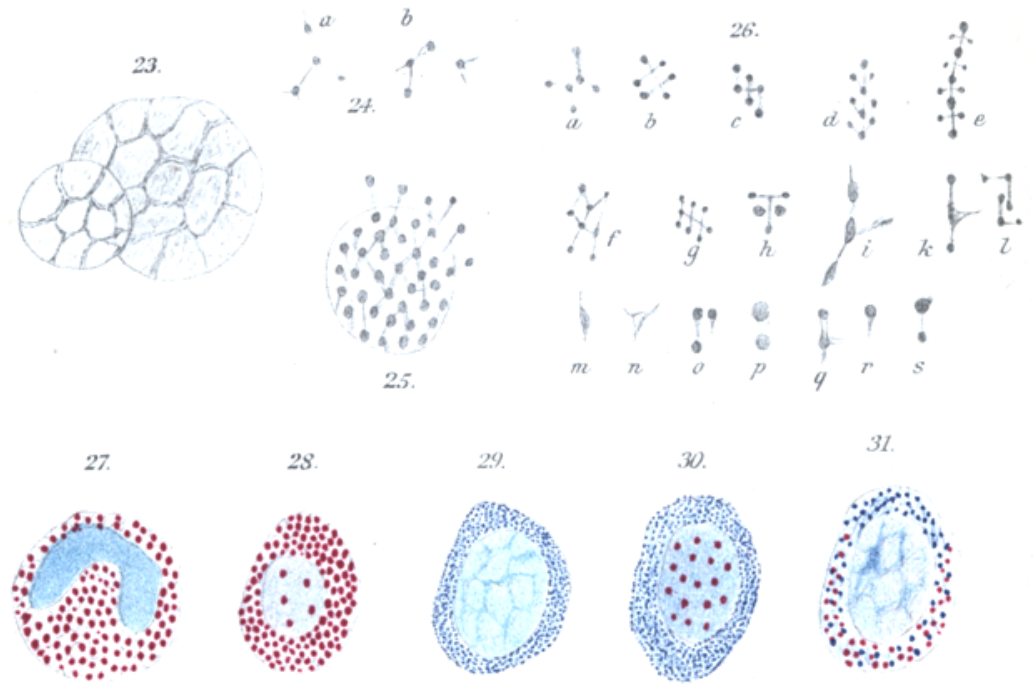

32.

33.

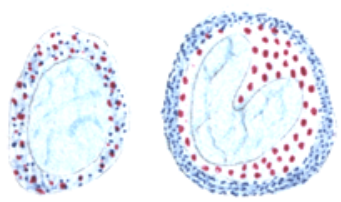

34.

35.

36.

37.
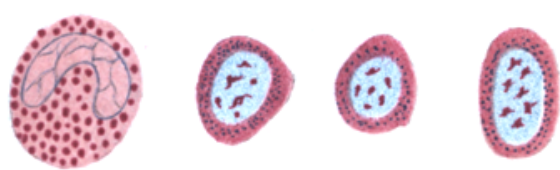

38.

39.

40.

42.

43.
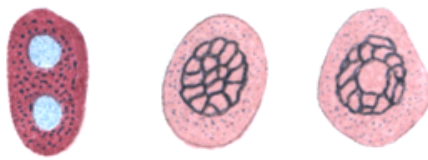

8

88
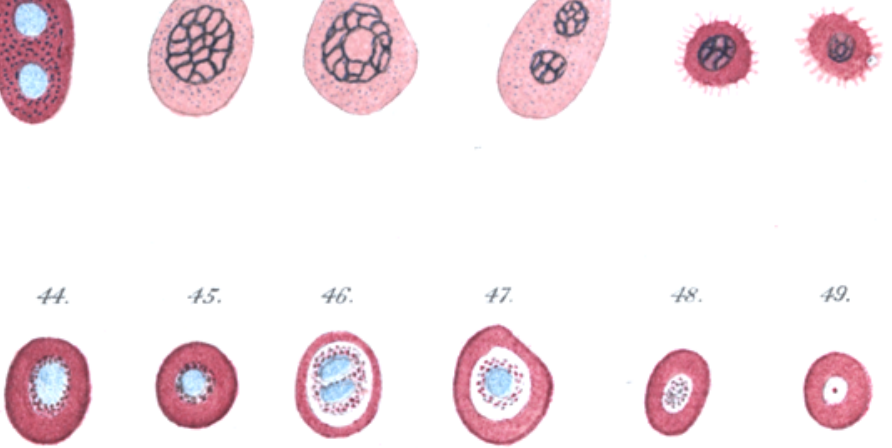

t8.

49.

W A. Meyn Fith Inst BertanS 
dass in der Erkenntniss der morphologischen und biologischen Verhältnisse des Knochenmarkes erst der Anfang gemacht ist.

I. Granulirung und Struktur der hämoglobinfreien Knochenmarkzellen.

In der eben citirten Arbeit habe ich die Mahnung ausgesprochen, bei derartigen Untersuchungen ja wicht mit der Anwendung einer Methode sich zu begnügen. Dementsprechend bediente ich mich auch dieses Mal verschiedener Conservirungs-, Einbettungs- und Färbemethoden. Die in Formaldehyd ( $4 \mathrm{pCt}$.) und Alkohol (von steigender Concentration) oder in Müller-Sublimat gehärteten Objecte wurden mit Celloidin oder Paraffin oder beiden Substanzen durchtränkt und von ihnen $3-10 \mu$ dicke Schnitte hergestellt; für das Studium gewisser Formen ist die Durchforschung nicht nur sehr feiner, sondern auch etwas dickerer Schnitte unentbehrlich.

Was die Färbemethoden anbelangt, so kann ich zunächst die am Schluss des oben erwähnten Beitrages mitgetheilte angelegentlich empfehlen. Auf feine Celloidinschnitte von Formolpräparaten lässt man 12 Stunden lang im Brütschrank eine Lösung von Süurefuchsin-Anilinöl (10:50) einwirken; dann werden dieselben rasch mit Wasser abgespült, durch Hämatoxylinlösung (Delafield) gefärbt und schliesslich mit Salpetersäurealkohol (1:100) differencirt; man kann aber auch die Hämatoxylinfärbung der Differencirung folgen lassen, nur darf diese dann eine weniger intensive sein. Bei Paraffinpräparaten verfährt man besser nach der ursprünglich von Altmann angegebenen Methode mit der Modification, dass man bei der Differencirung mit Pikrinsäurealkohol nicht erwärmt, sondern die aufgeklebten Schnitte für längere Zeit in eine kalte Lösung eintaucht und dann mit Hämatoxylin färbt.

Während man an den Objecten, welche nach der ursprünglich Altmann'schen Methode tingirt sind, meistens nur die Zellgranula, im Kern höchstens einige gefärbte Körner (Kernkörperchen?) nachweisen kann, treten bei den eben angegebenen Modificationen gewisse Granula als intensiv roth gefärbte Gebilde hervor, die chromatischen Bestandtheile des Kerns zeigen dagegen die Hämatoxylintinction; ausserdem finden sich zuweilen im Kern noch 
mehr oder weniger intensiv gefärbte rothe Körner. - Sehr auffallend ist namentlich an Formolpräparaten die grosse Zahl der granulirten Zellen, welche über die nicht granulirten zu überwiegen scheinen, ja an der Peripherie des Knochenmarkcylinders sind fast nur die ersteren vorhanden. Sie enthalten theils einfache, theils polymorphe, heller und dunkler gefärbte Kerne; ihre Protoplasmasäume sind bald breiter, bald etwas schmäler. Solche Präparate eignen sich sehr, um von dem grossen $W$ echsel in Bezug auf Grösse, Form, Zahl and Farbenintensität der Granula sich zu überzeugen. Ausser runden Granula trifft man stäbchen- und spindelförmige; andere scheinen durch Fortsätze unter einander in Verbindung zu stehen. Neben Zellen, welche vorwiegend grosse oder kleine Granula führen, fanden sich solche mit Körnern verschiedener Grösse und verschiedener Farbenintensität. Ich darf in dieser Hinsicht auf die früheren Mittheilungen verweisen; diese Verhältnisse haben daselbst eine ausführliche Darstellung erfahren. Es sei deshalb nur noch das gar nicht seltene Vorkommen von Mitosen in granulirten Zellen erwähnt, weil ein solches früher bezweifelt wurde.

Die angegebenen Methoden sind überdies wohl die einfachsten, um an granulirten Zellen die Existenz von. Sphären zu demonstriren. Dieselben erscheinen als lichte Gebilde, welche sich scharf gegen die Zellgranula abgreuzen und in ihrem Innern ein oder mehrere Körner enthalten. Eine Verwechselung mit Kernen ist durch die Hämatoxylinfarbe dieser ausgeschlossen. An nicht granulirten Zellen und Riesenzellen ist der Nachweis von Sphären bei Anwendung dieser Methode schwieriger; ebenso ergeben sich in Bezug auf Protoplasmastrukturen und die nicht chromatischen Bestandtheile der Kerne keine genügenden Aufschlüsse.

Um so lehrreicher sind in dieser Hinsicht die Bilder, welche man bei der Combination der Heidenhain'schen Eisenhämatoxylinmethode mit der Altmann'schen erhält. - Es ist sehr zweckmässig, die Präparate mit Anilinblau vorzufärben; in Folge der gelbgrünen Färbung, welche das Protoplasma annimmt, tritt der Zellleib, insbesondere auch seine Abgrenzung nach aussen deutlicher hervor. Nachdem man so vorbereitete Objecte den Proceduren des Heidenhain'schen Verfahrens unterworfen hat, 
übergiesst man sie mit Säurefuchsin-Anilinöl, erwärmt bis Dämpfe aufsteigen, und differencirt mit Pikrinsäurealkohol, ohne zu erwärmen, je nach Bedürfniss kürzere oder lüngere Zeit, jedenfalls aber so lange, bis die diffus rothe Färbung verschwindet und das Strukturbild wieder zum Vorschein kommt. Der Differencirungseffekt ist je nach der Dauer dieses Verfahrens und der Dicke der Schnitte, aber auch unter sonst gleichen Verhältnissen an verschiedenen Stellen und Zellen, auch wenn diese unmittelbar neben einander liegen, ein sehr ungleicher. Bedeutet dieses Ergebniss auf der einen Seite einen unverkennbaren Mangel der Methode, so erhält man andererseits gerade an solchen verschiedengradig differencirten Zellen sehr interessante Aufschlüsse über Protoplasma- und Kernstruktur der einzelnen Zellformen, namentlich, wenn man dieselben in verschiedenen Stadien der Differencirung einer Betrachtung unterzieht.

Hat man so lange differencirt, bis die diffuse Färbung eben verschwunden ist, so finden sich zahlreiche mehr oder weniger intensiv diffus gefärbte Kerne; an manchen derselben ist eine Struktur nicht nachweisbar, während an anderen, namentlich bei starker Durchleuchtung, noch Andeutungen einer solehen zu erkennen sind. Als ich zuerst auf eine solche diffuse Färbung der Kerne aufmerksam machte, war man eine Zeit lang geneigt, alle derartigen Kerne als in Degeneration begriffene aufzufassen. Wie vorsichtig man in der Verallgemeinerung einer solchen Annahme sein muss, geht daraus hervor, dass bei fortgesetzter Differencirung viele dieser Kerne den diffus vertheilten Farbstoff abgeben; es treten dann die fädigen und körnigen Bestandtheile mehr oder weniger intensiv gefärbt hervor; endlich verschwindet der Farbstoff auch aus diesen. Es vermögen also die verschiedenen Substanzen der Kerne den Farbstoff der Differencirungsflüssigkeit gegenüber verschieden lang zurück zu halten und es zeigen in dieser Beziehung die einzelnen Kerne sehr grosse Differenzen.

Hat man eine Differencirung bis zu dem Grade erreicht, dass der diffuse Farbstoff vollständig aus den Kernen verschwunden ist, dann kommen verschieden intensiv tingirte, rundliche, fädige und verzweigte Gebilde zum Vorscheiu, welche die Kerne dicht erfüllen (Taf. I. Fig. 1-7). Auf den ersten Blick haben solche 
Kerne grosse Aebnlichkeit mit in Theilung begriffenen. Die Anordnung der Fäden und Körner ist aber eine andere, es feblt, wie ein Vergleich mit sich theilenden Kernen an demselben Object lehrt, die diesem Stadium der mitotischen Theilung eigenartige Aufstellung der Fäden. Auch das Verhalten bei der weiteren Differencirung ist ein anderes; die mitotischen Kerntheilungsfiguren pflegen noch gefärbt $z u$ sein, wenn die anderen Kerne ihren Farbstoff schon vollständig abgegeben haben und die in ihnen gelegenen Fädchen und Körner eben noch als blaue Gebilde zu erkennen sind. In den grösseren Zellen, den Riesenzellen insbesondere, erhält man den Eindruck einer netzförmigen Anordnung mancher dieser Fäden (Taf. I. Fig. 14-18). Besonders bemerkenswerth ist das Verhalten der Kernwandschicht, welche bald als ein intensiv gefärbtes, membranartiges Gebilde sich darstellt, bald aus feineren Körnern und Fäden zu bestehen oder von solchen durchsetzt zu werden scheint. Manche Kernfäden inseriren sich an der Kernwandschicht; in Folge dessen entsteht noch mehr der Eindruck, als ob sie selbst aus Fäden aufgebaut wäre. In diesem Sinne ist noch ein anderer Befund verwerthbar. Bei manchen Zellen, namentlich bei solchen, deren Kerne im Zustand der Theilung sich befinden, besteht $z$ wischen der Kernwandschicht und der angrenzenden Protoplasmazone ein heller Raum, welcher von theils intensiv, theils schwach oder gar nicht gefärbten Fäden durchsetzt wird (Taf. I. Fig. 19 und 22). Dieselben hängen mit analogen Gebilden in der umgebenden Protoplasmazone zusammen. Ausserdem enthält der Leib mancher Zellen, der Riesenzellen insbesondere, rundliche, spindelförmige, verästigte und fädige Gebilde, welche aber meistens schwächer gefärbt sind (Eig. 14, 15, 17 und 19). In der Anordnung zeigen sie eine nicht zu verkennende Aehnlichkeit mit den Bildern, welche bei der Anwendung von Methylenblau im Zellleib sichtbar werden. Ich darf in dieser Hinsicht auf meine früheren Mittheilungen verweisen. Ich vermuthe deshalb, dass diese Körner und Fäden nicht identisch sind mit den oben beschriebenen Granula, denn diese pflegen auch bei dieser Methode intensiv gefärbt zu sein und den Farbstoff lange festzuhalten. Sehr viele Knochenmarkzellen, namentlich die kleineren Formen, sind mehr oder weniger scharf begrenzt, bei den grösseren aber, 
namentlich manchen Riesenzellen, treten von der Zellperipherie zahlreiche feine, bald intensiver, bald schwächer oder gar nicht gefärbte Ausläufer ab, welche aus einem dichten, an der Peripherie der Zelle gelegenen Netz von Fibrillen abzweigen (Fig. 17 and 18).

Ein weiterer Vorzug dieser mit Eisenlack gebeizten Präparate ist der, dass an den Riesenzellen die Sphären kenntlich werden (Fig. 20, 21, 22). Es haben diese durch M. Heidenhain ${ }^{1}$ ) eine ausführliche Darstellung erfahren; ich will mich deshalb nur auf einige kurze Bemerkungen beschränken. In manchen Riesenzellen habe ich in einer Sphäre vier oder mehr Centralkörper wahrgenommen, welche zuweilen eine noch beträchtlichere Differenz in der Grösse aufweisen, als dies Heidenhain erwähnt. Eine so grosse Zahl von Centralkörpern, wie dieser Autor, habe ich nicht beobachtet, ohne deshalb ein solches Vorkommen leugnen zu wollen. Neuestens hat allerdings Niesing ${ }^{2}$ ) den Verdacht ausgesprochen, dass nicht alle Körnchen, welche Heidenhain als Centralkörper auffasst, diese Deutung verdienen; er will als solche nur diejenigen anerkennen, welche die Ursprungspunkte für die Protoplasmafibrillenstrahlung abgeben. Niesing konnte einzelne centrirte Fibrillen bis an die Zellgrenze verfolgen. Für andere nimmt er eine Endigung an der.Kernhaut an. Im Gegensatz zu Heidenhain hat er bei Abhebungen des Protoplasmas vom Kern feinste, vòn dem Centralkörper nach dem Kern gehende, Brücken gesehen. Auch auf die bedeutungsvollen, diesen Gegenstand betreffenden Beobachtungen Reinke's ${ }^{3}$ ) darf ich nicht unterlassen, schon an dieser Stelle hinzuweisen. - In Fig. 20 habe ich eine Riesenzelle abgebildet; in der Mitte der complicirten Kernfigur liegen vier Centralkörper, von denen eine feine, gegen die Kernwand gerichtete Strahlung ausgeht. Die in Fig. 21 und 22 dargestellten Riesenzellen enthalten vielgestaltige Kerne. In jedem der hellen Felder, welche von den Bändern der complicirten Kernfigur umschlossen werden, ist ein feines Korn gelegen, von dem feine, gegen die Kernwand verlaufende, Strahlen

1) M. Heidenhain, Neue Untersuchungen über die Centralkörper u. s.w. Archiv f. mikroskop. Anat. Bd. 43. 1894.

2) Niesing, Zellstudien. Archiv f. mikroskop. Anat. Bd. 46. 1895.

3) Reinke, Zellstudien. Ebendaselbst, Bd. 43 und 44. 1894. 
abtreten. Ich habe derartige Verhältnisse nur an Riesenzellen gefunden, deren Kerne eine sehr complicirte Architectur aufwiesen. Ist die Zahl der Riesenzellen, wie das im rothen Knochenmark des Kaninchens vorkommt, eine sehr beträchtliche, so wird man fast immer einzelne solche, mit mehreren Sphären ausgestattete, Formen treffen; die Beziehung dieser Gebilde zur Kernmembran lässt meines Erachtens kaum eine andere Deutung $\mathbf{z u}^{1}$ ). Jedenfalls stehen sie zur 'Theilung der Kerne der Riesenzellen in Beziehung. Bei dem derzeitigen Stand dieser Frage scheint mir ein solcher Nachweis besonders bedeutungsvoll. Im Anschluss daran will ich noch des Vorkommens von mitotischen Kerntheilungsfiguren, welche in die Körper der Riesenzellen eingeschlossen sind, indem ich auf meine früheren Mittheilungen Bezug nehme, Erwähnung thun (Fig. 18).

Durch die Untersuchungen Altmann's, Gaule's, Ogata's, Lukjanow's, sowie diejenigen Heidenhain's, Reinke's, Schloter's u. A. ist die bedeutungsvolle Thatsache zu Tage gefördert, dass der Kern ausser dem Chromatin (Basichromatin) noch andere morphologische Substanzen in seinem sog. Saft einschliesst, welche tinctoriell von dem Basichromatin wesentlich sich unterscheiden; ich meine die cyanophile Körnelung Altmann's, das Oxychromatin Heidenhain's und das Oedematin Reinke's. Inwiefern diese Körper different oder identisch sind, lässt sich heute noch nicht mit Bestimmtheit sagen. Schloter ist der Ansicht, dass die cyanophile Körnelung Altmann's und

1) In seiner bekannten Arbeit über Theilung und Kernformen bei Leukocyten und über deren Attractionsspbären erwähnt Flemming, dass ich zuerst an ringförmigen Kernen der Leukocyten auf das Vorkommen eines glänzenden Korns und einzelner lichter Fäden in der Mitte der bellen Felder hingewiesen, aber in den Kern selbst verlegt habe. Die Wabrnehmung, dass die Fäden sich an der Kernmembran, welche an solchen Stellen wie ansgezackt sein kann, inseriren, war für mich die Veran]assung gewesen, eine Beziehung zwischen diesen Gebilden und der Kernwandscbicht anzunehmen. In Anbetracht der oben mitgetheilten Thatsachen wird man einräumen müssen, dass diese Vorstellung keine ganz irrthümliche war. Auch complicirte Kernfiguren, welche helie Felder mit central gelegenen Körnern umschliessen, babe ich früher bereits abgebildet. (Weitere Mittheilungen über Kern- und Zelltheilungen. Archiv f. mikrosk. Anat. Bd.31.) 
das Oedematin Reinke's dieselben Granulaarten bedeuten. Auch in der Zellsubstanz werden ausser den Granula Altmann's oxyplasmatische und achromatische Granulationen angenommen, zu denen dann noch die in den früheren Mittheilungen beschriebenen acidophilen, neutrophilen und basophilen Ehrlich's hinzukämen. Auch für diese Granula der Zellsubstanz gilt das oben Gesagte, dass es zur Zeit unmöglich ist zu entscheiden, inwieweit sie verschiedenen Arten entsprechen, ob ihnen eine verschiedene chemische Constitution zukommt, ob sie in einander übergehen können und welche functionelle Bedeutung sie haben. Die oben mitgetheilten Thatsachen weisen darauf hin, dass manche der im Kern und Zellleib gelegenen Substanzen denselben Farbstoffen gegenüber unter wechselnden Verhältnissen ein verschiedenes Verhalten darbieten und dass die Dauer der Einwirkung der differencirenden Flüssigkeit eine grosse Rolle spielt. Wir haben die Erfahrung gemacht, dass Gebilde, welche bei dem ursprünglichen Altmann'schen Verfahren nicht gefärbt erscheinen, nach vorausgegangener Behandlung mit Hämatoxylineisenlack den Farbstoff verschieden lange festzuhalten vermögen. Daraus könnte viel eher auf differente physikalische, als auf chemische Eigenschaften geschlossen werden.

Eines der interessantesten mit diesen Methoden gewonnenen Ergebnisse ist zweifellos der Nachweis des Zusammenhanges zwischen den Substanzen des Kerns und Zellleibes. Es stimmen meine Befunde in vielfacher Hinsicht mit denjenigen Niesing's, namentlich aber Reinke's überein. Der Letztere erörtert die Frage, ob die Fäden aus dem Kern durch Poren der Kernmembran in den Zellleib übertreten oder ob nur ein Zusammenhang durch die in der Kernwandschicht gelegenen Fäden vermittelt wird. Den oben berichteten Beobachtungen zu Folge und meiner Vorstellung über die Zusammensetzung der Kernwandschicht aus Fäden gemäss möchte ich der letzteren Anschauung den Vorzug geben und befinde mich auch in dieser Hinsicht mit den Auffassungen in Uebereinstimmung, welche Reinke ${ }^{1}$ ) neuestens vertreten hat. Auf die Beziehungen zwischen Sphäre,

1) Vergl. Reinke, Zellstudien. 2. Th. Archiv f. mikr. Anat. Bd. $44-$ Waldeyer's Bericht. Berl. klin. Wochensehr. 1895. Schriftliche Mit. theilung. 
Kern und Substanz des Zellleibes ist bereits oben hingewiesen worden.

Der bedeutungsvolle Wandel, welcher sich in den Anschauungen über die Struktur des Protoplasmas und die Beziehungen zwischen den Substanzen des Zellleibes und des Kerns vollzieht, ermuthigt mich, ähnliche Beobachtungen in Erinnerung zu bringen, über welche vor nach unseren Begriffen sehr langer Zeit von mir berichtet worden ist. Ich habe damals nicht nur an den Ganglienzellen (1865-1867), sondern auch an den Knorpelzellen (1875) eine fibrilläre Beschaffenheit des Protoplasmas, sowie einen Zusammenhang zwischen den Fäden des Kerns und denjenigen des Protoplasmas beschrieben. In einer späteren Arbeit ${ }^{1}$ ) versuchte ich nachzuweisen, dass diese Anordnung eine bei den verschiedensten Zellen vorkommende, also weit verbreitete sei. Ich unterliess nicht, zu betonen, dass man die Fäden im Kern und Protoplasma nicht nur an gehärteten, sondern auch an überlebenden Objecten wahrnehmen könne und dass an isolirten Kernen diese Fäden über die Peripherie vortreten, event. mehr oder weniger weit in das Protoplasma hinein sich fortsetzend. Daselbst sind nicht nur meine eigenen Beobachtungen, sondern auch die Angaben Anderer über diesen Gegenstand verzeichnet. Es haben diese Mittheilungen wenig Beachtung und noch weniger Anklang gefunden. Als man später mehr geneigt war, die Protoplasmafibrillen anzuerkennen, hat man gerade aus dem von mir behaupteten Zusammenhang der Kernfäden mit den Protoplasmafäden deduciren wollen, dass die von mir beschriebenen Gebilde mit den jetzt zur Anerkennung gelangten nicht identisch seien. Aus den oben mitgetheilten und weiter unten noch auszuführenden Thatsachen geht hervor, dass zwischen den Gebilden des Kerns und des Zellleibes innige Beziehungen bestehen; aber wenn dem auch nicht so wäre, ich kann die Berechtigung einer solchen Beweisführung nicht anerkennen. Ich füge noch hinzu, dass diese Wahrnehmungen für mich die Veranlassung gewesen waren, die damals für die Zelle geläufige Definition ${ }^{2}$ )

1) J. Arnold, Ueber feinere Struktur der Zellen unter normalen und pathologischen Bedingungen. Dieses Arcbiv. Bd. 77. 1879.

2) Der betreffende Passus lautet wörtlich: „In Anbetracht dieser Erfahrungen muss es als fraglich bezeichnet werden, ob die seit Max 
als unzureichend zu kennzeichnen. Hoffentlich verstosse ich nicht zu sehr gegen den Geist und Geschmack unserer Zeit, wenn ich für diese Mittheilungen, welche erst in $Z$ weifel gezogen, dann umgedeutet und schliesslich ignorirt wurden, Berücksichtigung erbitte.

In der Lehre der Protoplasmastrukturen ist eine der schwierigsten Fragen, die nach der Existenz von gesonderten Fibrillen und der Lage der Granula zu diesen, bezw. zu den anderen Bestandtheilen des Zellleibes. Ich kann auf die Literatur dieses Gegenstandes an dieser Stelle nicht eingehen; die neveren Arbeiten Altmann's, Bütschli's, Flemming's, Heidenhain's, Reinke's u. v. A. sind ja allgemein bekannt; überdies darf ich auf die zusammenfassenden Darstellungen und Berichte von Flemming, Hertwig, Waldeyer u. A. verweisen. - Da es auch an den feinsten Schnitten unmöglich ist, über die eben berührten Verhältnisse Aufschlüsse zu erhalten, machte ich Versuche mit Isolirung der Zellen. Legt man möglichst kleine Stückchen in 1procentiger Osmiumsäure, schüttelt und färbt dieselben nach 6 Stunden mit Ehrlich'scher EosinHämatoxylinlösung, dann findet man zahlreiche vollkommen isolirte Zellen, an welchen ganz deutliche Körner und Fäden zu erkennen sind. Betreffs der Beziehungen dieser zu einander ge. langt man aber auch zu keiner sicheren Ueberzeugung. - Sehr überraschende Resultate erbielt ich dagegen bei der Anwendung folgenden Verfahrens. Man schiebt den Markcylinder mittelst eines feines Glasstabes aus seinem knöchernen Schaft soweit heraus, dass man von dem ersteren kleine Stückchen mit der Scheere abschneiden und in kleine, mit 10procentiger Jodkalilösung gefüllte, gut schliessende Gläschen übertragen kann. Wenn nach 12 Stunden die Knochenmarkpartikelchen gallertig geworden sind, legt man sie in 1procentige wässrige Eosinlösung, in welcher

Schultze geläufige Definition der Zelle ausreichend ist, da sie diese wesentlichen Strukturverbältnisse unberücksichtigt lässt. In einer solchen muss meines Erachtens zum tnindesten das ausgesagt werden, dass die Zellen aus Kern und Belegungsmasse bestehen, welche beide in einer homogenen Grundsubstanz Körner und Fäden eingebettet enthalten." (Der Schlusssatz ist auch im Original gesperrt gedruckt.) 
sie 3 Stunden und länger verbleiben. Die gefärbten Objecte kann man dann jeder Zeit einer Besichtigung unterziehen, indem man sie mit einem möglichst dünnen Deckglas, das an der einen Seite unterstützt.wird, deckt, und eine Verdunstung durch Vaselinverschluss hintanhält.

An solchen Objecten ist die Zwischensubstanz theils gequollen, theils gelöst und eine mehr oder weniger vollständige Isolirung der Zellen erreicht. Ich sehe ab von einer Beschreibung der prall gefüllten Fettblasen, sowie der in verschiedenem Zustand der Füllung mit Fett befindlichen kleinen und grösseren Zellen, ebenso der spindligen und verästigten Formen, welche oft sehr lange und zahlreiche, mit eigenthümlichen Anschwellungen versehene Ausläufer entsenden; uns interessiren hier nur die verschiedenen Leukocytenarten und eigentlichen Markzellen. Manche derselben sind nach Form und Struktur ganz gut erhalten; man sieht deutlich netzförmig angeordnete Fäden im Kern, welche zum Theil an die Kernmembran sich ansetzen und feinere, sowie etwas gröbere Granula führen. Bei längerer Einwirkung wird der Kern lichter, blasig und die gröberen Fäden mit ihren Granulis treten aus demselben hervor (Fig. 23 und 24). Aehnliche Veränderangen vollziehen sich auch am Protoplasma. Zuerst sind die von Fäden und Körnern durchsetzten Leiber der Zellen ganz gut erhalten, nach einiger Zeit lösen sich von der Oberfläche einzelne drehrunde Fäden $a b$ und stehen frei über die Zellgrenze vor (Fig. 25). Weiter kommt es zur Befreiung ganzer Systeme von Fäden von sehr verschiedener Länge, Dicke, Lichtbrechung und Abgrenzung. Was zunächst die letztere anbelangt, so erscheinen viele Fäden drehrund und scharf abgegrenzt, so namentlich die peripherisch gelagerten; bei anderen ist die Abgrenzung weniger scharf und die runde Form weniger ausgesprochen, so dass man an Bruchstücke von Scheidewänden denken könnte. Manche dieser Fäden sind dicker, andere dünner, die einen stark lichtbrechend, die anderen mehr hyalin. Derselbe Wechsel besteht bezüglich der Farbenintensität; neben intensiver tingirten, trifft man schwach oder gar nicht gefärbte. Ein ganz ähnliches Verhalten bieten die Granula dar, so dass man allerdings den Eindruck erhält, als ob man es mit verschiedenen Arten von Fäden and Granula zu thun hat. Betreffs 
der Beziehung der ersteren zu den letzteren kann man sich auf das Bestimmteste davon überzeugen, dass viele Granula in den Fäden selbst liegen, deren Verlauf gleichsam unterbrechend. Sind die Granula sehr zahlreich, so erhält man mehr den Eindruck von aus Granulis und stäbchenförmigen Bindegliedern bestehenden Ketten (Fig. 26). Eine derartige Verbindung hat aber nicht nur in einer, sondern in zwei und vielleicht in mehreren Richtungen statt, wodurch eine netzförmige Anordnung zu Stande kommen kann (Fig. 26). Neben solchen wirklich netzförmigen Verbindungen kommen aber auch Kreuzungen der Fäden und Fadensysteme vor, so dass es manchmal den Anschein hat, als ob zwei netzförmig angeordnete Fadensysteme derart durch einander geschoben wären, dass ihre Bindeglieder sich kreuzten (Fig. 26). Wenn ich nicht irre, so hängen nicht nur Granula von derselben Grösse, Lichtbrechung und Farbenintensität durch Bindeglieder unter einander zusammen, sondern es kommen in denselben Fäden Granula von verschiedener Qualität, jedenfalls von verschiedener Grösse, vor. $O b$ es auch freie, zwischen den Stäbchen gelegene Granula giebt, war ich nicht im Stande mit Sicherheit $\mathrm{zu}$ ermitteln. Selbstverständlich trifft man solche in grosser Zahl; sehr viele derselben sind aber nicht kreisrund, sondern haben einen oder mehrere, sich zuweilen verjüngende Fortsätze. Besonders deutlich ist das bei den grossen Körnern vieler eosinophiler Zellen. Ich verweise auf die entsprechenden Befunde an Schnittpräparaten. Andere Male entsteht das Bild frei in den Maschen $z$ wischen den Bindegliedern gelegener Körner dadurch, dass der lichte Verbindungsfaden durch einen anderen, denselben kreuzenden verdeckt wird. Ungeachtet dieser eben aufgedeckten Quellen der Täuschung soll das Vorkommen freier Körner nicht geleugnet werden. Ausserdem ist wohl noch eine zwischen den Fäden und Körnern gelegene Substanz anzanehmen.

Die grosse Bedeutung dieser Befunde für unsere Anschauungen über Protoplasmastrukturen liegt auf der Hand. Wenn ich auf eine Erörterung derselben an dieser Stelle verzichte, so geschieht das in der Ueberzengung, dass erst durch nach denselben Methoden angestellte Untersuchungen an anderen Objecten - derartige Erfahrungen stehen mir bereits zu Gebot - ein 
Beweismaterial geschaffen werden muss, an der Hand dessen über die hier in Betracht kommenden schwierigen Fragen eine Entscheidung getroffen werden kann. Nur das möchte ich hervorheben, dass den oben berichteten Thatsachen, namentlich ihrer Beziehung zu den Fäden und Bindegliedern zu Folge viele Granula, unter ihnen namentlich die eosinophilen, nicht als von aussen aufgenommene corpusculäre Zelleinschlüsse, sondern als der Zelle zugehörige, vielleicht durch Umwandlung der Substanz derselben entstandene Gebilde betrachtet werden müssen, selbstverständlich ohne sie in dem ursprünglich Altmann'schen Sinne als bioplastische Einheiten der Zelle ausgeben zu wollen. Die oben berichteten Thatsachen sind ja wichtige Belege dafür, dass die Struktur des Protoplasmas eine viel complicirtere ist.

Waldeyer betont in seinem neuesten Bericht mit Recht, dass es verfrüht wäre, den einzelnen Bestandtheilen des Zellleibes schon bestimmte functionelle Rollen zuzutheilen. Andererseits möchte ich darauf hinweisen, dass doch eine Reihe von Thatsachen vorliegen, welche auf eine Beziehung gewisser Granula und Fäden zum Stoffwechsel hindeuten. Ich erlaube mir zunächst in dieser Hinsicht eine ältere, wenig berücksichtigte Beobachtung von mir in Erinnerung zu bringen. I $\operatorname{ch}^{1}$ ) habe nachgewiesen, dass bei der Infusion von indigschwefelsaurem Natron in das Blut lebender Thiere nicht nur an der Peripherie der Knorpelzellen, sondern auch im Inneren derselben, sowie im Kern blau gefärbte Fäden und Körner zum Vorschein kommen. Sehr interessant sind ferner in dieser Hinsicht die mit Methylenblaufärbung an lebenden Zellen angestellten Versuche, bei denen sich gewisse Granula tingiren (Schultze, Kowalewsky, Mitrophanow, Hausmann). Andererseits gehören auch hierher die Veränderungen, wie sie namentlich Nissel durch seine gründlichen Untersuchungen an den Ganglienzellen unter verschiedenen Verhältnissen aufgedeckt hat. Ein sehr wichtiger Fingerzeig, welche Bedeutung solchen Strukturverhältnissen unter pathologischen Bedingungen zukommt.

1) J. Arnold, Ueber die Abscheidung indigschwefelsauren Natrons im Knorpelgewebe. Dieses Archiv. Bd.73. 1878. 
II. Die Granulirung hämoglobinhaltiger Kuochenmarkzellen.

Einer Kategorie von Zellen ist bisher keine Erwähnung geschehen, der kernhaltigen hämoglobinführenden. Zur Herstellung von Schnittpräparaten empfiehlt sich am meisten die Formolhärtung und die Tinction mittelst Säurefuchsin-Hämatoxylin. Als Differencirungsmittel ist in diesem Falle der Pikrinsäurealkohol nur dann vorzuziehen, wenn man das eigenthümliche Verhalten der Blutkörper, welche theils gelb, theils gelbroth gefärbt werden, bei der Differencirung prüfen will. Die Zahl der kernhaltigen rothen Blutkörper ist im rothen Koochenmark gewöhnlich eine ziemlich beträchtliche, noch grösser, wenn man den Thieren Blut entzogen oder diese nach vorausgegangener Nahrungsentziehung aufgefüttert hat.

Es sind die bekannten, bald kleineren, bald grösseren Formen mit schmalen, zuweilen gezackten oder strahlig auslaufenden (Fig. 42 und 43) Protoplasmasäumen und dunklen Kernen, in denen bei ausgiebiger Differencirung sehr dichte Fadensysteme zum Vorschein kommen; andere Male erscheinen diese auch unter solchen Verhältnissen mehr homogen oder sie lassen im Inneren einen eigenthümlichen hellen Fleck erkennen. Die Kerne sind bald grösser, bald kleiner, liegen mehr central oder excentrisch oder ragen über den Contour der Zelle hervor. Die gewöhnlich runden Kerne können eingeschnürt sein oder eine gelappte Form annehmen ${ }^{1}$ ). Die Färbung des Protoplasmas ist eine in Bezug auf Intensität und Vertheilung wechselnde; manche Zellen haben ein mehr geflecktes Aussehen. Da ich mir sagen musste, dass der Wechsel im Gehalt an Hämoglobin, wie er an solchen Präparaten zum Ausdruck gelangt, namentlich die eben erwähnte ungleiche Vertheilung eine Folge der Conservirungsmethode sein könnte, habe ich Trockenpräparate hergestellt, an denen betreffs des Hämoglobingehaltes wohl die sichersten Aufschlüsse zu erwarten sind. Färbt uan dieselben mit Hämatoxylin-Eosin so erhält man im Wesentlichen dieselben Resultate.

1) Man vergleiche auch die Mittheilungen von Ti m of ejewsk y (Centralbl. f. allgem. Path. 1895. No.3/4) über die verschiedenen Formen der Kerne. 
Sehr interessante Befunde ergaben sich an Trockenpräparaten, welche mit Eosin-Methylenblau gefärbt wurden. Ich verfuhr dabei in folgender Weise. Die Plättchen wurden 12 Stunden in gesättigte alkoholische, 3 Stunden in wässrige 1 procentige Eosinlösung gelegt. Nachdem sie rasch mit Wasser abgespült worden waren, übergoss ich sie mit Löffler's Methylenblaulösung, erwärmte bis zur Entwickelung von Dämpfen, spülte dann wieder rasch mit Wasser ab und differencirte schliesslich mit Alkohol. Auch hier ist es zweckmässig, in verschiedenem Grade zu differenciren. Die letzte Differencirung nahm ich unter dem Mikroskop mit Origanumöl vor.

An solchen Präparaten fallen zunächst 2 Arten von granulirten Zellen auf, erstens solche mit theils feineren, theils gröberen, rothen Granula, deren Kerne hellblau gefärbt sind, und zweitens solche mit sehr feiner blauer Granulirung, in denen die bellblau oder manchmal mehr hellblauroth gefärbten Kerne rothe Granula enthalten (Fig. 27-30). Neben diesen beiden Haupttypen mit rother oder blauer Granulirung des Protoplasmas finden sich solche, welche rothe und blaue Granula zugleich enthalten, wie ich das in meiner früheren Mittheilung schon hervorgehoben habe (Fig. 31-33). Die Vertheilung dieser ist zuweilen eine eigenthümliche, in der Art, dass die blauen Granula mehr die eine, die rothen die andere Seite des Zellleibes einnehmen, die rothen näher dem Kern, die blauen mehr an der Zellperipherie liegen; andere Male sind beide Arten mehr durch einander geworfen, oder dieselben Granula sind theils roth, theils blau gefärbt. Das letztere Verhalten ist wohl nur der Ausdruck einer mangelhaften Differencirung; auch der Befund von rothen und blauen Granula in derselben Zelle kann in diesem Sinne gedeutet werden, insofern, es sich um gleich grosse Granula handelt. Wenn aber in derselben Zelle die grossen Granula ausschliesslich roth, die kleinen blau gefärbt sind, so darf daraus schon eher auf das Vorhandensein verschiedener Arten geschlossen werden, ein Vorkommen, das ja auch bei den Isolirungsversuchen als wahrscheinlich sich herausgestellt hat. Dass aus diesen Befunden noch nicht auf eine Umwandlung der verschiedenen Granulaarten in einander geschlossen werden darf, ist selbstverständlich ${ }^{1}$ ).

1) Ausser den früber erwähnten Autoren gedenkt auch Sachar off (Arch. Archiv f. pathol. Anat. Bd.144. Hft. 1. 
Das interessanteste Ergebniss an diesen Plättchen war aber der Befund von grossen, mit rothen Granula erfüllten Zellen, deren Leib diffus roth gefärbt war und deren Kerne zuweilen im Zustande der Mitose sich befanden (Fig. 34), sowie das Vorkommen zahlreicher hämoglobinhaltiger, gekurnter Zellen von dem Aussehen kernhaltiger rother Blutkörper, welche im Zellleib noch blaue Granula führten (Fig. 35-38). - Die Kerne zeigten das oben beschriebene Verhalten; an der Grenze gegen das umgebende Protoplasma lagen rothe Granula, so dass sie von diesem durch eine solche Zone geschieden schienen (Fig. 44-47). Nach der Anordnung des Hämoglobins und der Kerne muss man vermuthen, dass diese Formen mit den gewöhnlichen kernhaltigen rothen Blutkörpern, wenn nicht identisch, so doch nahe verwandt oder Vorstufen derselben sind. Verschiedenheiten sind gegeben durch die basophile Granulirung des Zellleibes. und die acidophile des Kerns, bezw. der Kernperipherie. Des Vorkommens der letzteren in Hämatoblasten thut auch Sacharoff Erwähnung; er stellt sich vor, dass dieselben ausgestossen werden und zur Entstehung der eosinophilen Zellen Veranlassung geben.

Welche Bedeutung haben die eben berichteten Thatsachen für unsere die Provenienz der rothen Blutkörper betreffenden Vorstellungen? Zwei Ansichten stehen sich zur Zeit als die herrschenden unvermittelt gegenüber. Der einen zufolge sollen die Leukocyten und Erythrocyten getrennte und zwar genügend gekennzeichnete Vorstufen - Leukoblasten und Erythroblasten - haben, von denen die ersteren nur nach dem Typus der Amitose, die anderen nur noch demjenigen der Mitose sich theilten. So verlockend diese Hypothese ist und so grossen Anklang sie gefunden hat, so darf man sich andererseits doch nicht verhehlen, dass sie ihrer Hauptstütze beraubt worden ist, weil an einem Vorkommen mitotischer Kerntheilungsvorgänge auch bei Leukocyten heute nicht mehr gezweifelt werden darf. Einige darauf sich beziehende Beobachtungen wurden $\mathrm{ja}$ auch oben mitgetheilt. Dass dessen ungeachtet diese Anschauung nicht als widerlegt be-

f. mikroskop. Anat. Bd. 45) des Vorkommens basophiler Körnelung in eosinophilen Zellen. 
zeichnet werden kann, ist selbstverständlich; denn es muss die Möglichkeit zweier getrennter Vorstufen auch dann zugegeben werden, wenn beide nach demselben Typus sich theilen; aber das sicherste Unterscheidungsmerkmal ist hinfällig geworden. Dem gegenüber hat die ältere Ansicht, der zu Folge die kernhaltigen rothen Blutkörper aus einer Umwandlung ron Leukocyten entstehen, neuerdings wieder Vertreter gefunden. Müller, Wertheim u. A. ${ }^{1}$ ) beschreiben solche Uebergangsformen; ob sie als solche genügend charakterisirt sind, wird allerdings als fraglich bezeichnet; dieselben Strukturverhältnisse findet man bei den meisten Markzellen, welche nach dem Typus der Mitose sich vermebren. - Bei diesem Stand unserer Kenntnisse verdienen die oben beschriebenen Befunde - das Vorkommen cosinophiler Zellen, welche diffus vertheiltes Hämoglobin führen, sowie dasjenige basophiler Granulirung im Leib, acidophiler im Kern hämoglobinhaltiger Formen und endlich der kernhaltigen rothen Blutkörper mit verzweigtem Zellleib - Beachtung. Was zunächst die letzteren anbelangt, so sind die Bilder dieselben, wie bei den Zellen, welche im Zustande der amöboiden Bewegung fixirt wurden (Fig. 42 und 43). Vorausgesetzt, dass diese Deutung richtig ist, so fiele ein weiterer Unterschied zwischen Leukoblasten und Erythroblasten weg. Eine Beziehung zwischen den kernhaltigen hämoglobinführenden Zellen und den Leukocyten kann aber ferner in dem Vorkommen derselben Granula gefunden werden, wie sie im Zellleib der grossen sog. Markzellen zu treffen sind. In demselben Sinne liesse sich auch der Nachweis hämoglobinführender eosinophiler Zellen deuten; sie als degenerirende Formen anzusprechen (Sacharoff), dünkt mir mit Rücksicht auf das Vorhandensein mitotischer Kerntheilungsfiguren nicht zulässig. Das Auftreten eosinophiler Körner an der Peripherie der Kerne der rothen Blutkörper ist vielleicht auf einen Austausch der Granula des Zellleibes zu beziehen, welcher mit der später erfolgenden Metamorphose des Kerns in Zusammenhang steht (Fig. 44-48). Dass diese eosinophilen

1) Bezüglich der Literatur verweise ich auf die Arbeiten Loewit's, Müller's, van der Stricht's, Engel's, sowie namentlich auf den zusammenfassenden Bericht von $0 \mathrm{ppel}$ (Centralbl. f. allgem. Pathol. Bd. III). 
Granula, wie Sacharoff meint, ausgestossen werden und auf diese Weise die eosinophilen Granula der Leukocyten entstehen, ist mir mit Rücksicht aúf die oben beschriebene Form, Grösse und namentlich die Lage der Granula zu den Fäden, sowie auf den Befund solcher Granula in manchen kernlosen rothen Blutkörpern nicht wahrscheinlich. Die ganze Metamorphose der Kerns und Protoplasmas bei der Umgestaltung der kernhaltigen rothen Blutkörper in kernlose, sowie der dabei erfolgende Abbau des Kerns sprechen meines Erachtens dagegen. Ich begnüge mich mit diesen Andeutungen, weil diese Verhältnisse in der folgenden Mittheilung eine ausführliche Darstellung erfahren sollen.- Es sei deshalb schliesslich nur noch einmal Folgendes hervorgehoben: die eben berichteten Thatsachen, namentlich der Befund derselben Granulaarten in hämoglobinhaltigen and in hämoglobinlosen Zellen des Knochenmarks weisen auf eine genetische Beziehung beider Formen, deren Wesen wohl in einer Umwandlung des letzteren in die ersteren erblickt werden darf, hin. - Für die Annahme, dass nur bestimmte Arten hämoglobinloser Knochenmarkzellen einer solchen Leistung, bezw. Metamorphose fähig sind, so zulässig diese erscheinen mag, liegen zur Zeit genügende Anhaltspunkte nicht vor. Nur so viel steht fest, dass vorwiegend mitotische Theilungsprodukte hämoglobinfreier Zellen als Vorstufen der kernhaltigen rothen Blutkörper anzusehen sind. Damit soll nicht in Abrede gestellt werden, dass noch andere Entstehungsweisen dieser - Theilung von Zellen, welche bereits Hämoglobin führen, z. B. kernhaltiger rother Blutkörper - vorvorkommen können.

\section{Erklärung der Abbildungen.}

\section{Tafel I und II.}

Sämmtliche Figuren sind mit Zeiss Apochromat 2,0 mm und Ocular 8 oder 12 . Fig. 1-7. Knochenmarkzellen, gefärbt mit Eisenlack-Säurefuchsinpikrin; theils Formol-, theils Müller-Sublimathärtung.

Fig. 8-13. Eosinopbile Zellen; Färbung dieselbe. 8-12 Mäller-Sublimathärtung, 12-13 Formolbärtung.

Fig. 14 und 15. Grosse Zellen aus dem Knochenmark; Formolpräparat, Färbung dieselbe. 
Fig. 16-22. Riesenzellen aus dem Knochenmark; theils Formol-, theils Müller-Sublimatbärtung, Färbung dieselbe.

Fig. 23. Isolirte Knochenmarkzelle; Jodkalipräparat.

Fig. 24. Isolirte Fäden des Kerns; Jodkalipräparat.

Fig. 25. Eosinophile Zellen; Jodkalipräparat.

Fig. 26. Isolirte Fãden und Körner des Protoplasma; Jodkalipräparat.

Fig. 27 und 28. Zellen des Knochenmarks mit sog. acidopbiler Granulirung; Trockenpräparat; Eosin-Methylenblau.

Fig. 29 und 30. Zellen des Knochenmarks mit sog. basophiler Granulirung; Trockenpräparat; Eosin-Methylenblau.

Fig. 31-33. Knochenmarkzellen mit verschieden gefärbten Granula; Trockenpräparat; Eosin-Methylenblau.

Fig. 34. Eosinophile Zelle des Knochenmarks mit diffuser Hämoglobinfärbung; Trockenpräparat; Eosin-Hämatoxylin.

Fig. 35-38. Hämoglobinbaltige Zellen des Knochenmarks mit blauer Granulirung des Zellleibes und rothen Granula im Kern; Trockenpräparat; Eosin-Methylenblau.

Fig. 39-41. Hämoglobinbaltige Zellen des Knochenmarks in mitotischer Theilung; Trockenpräparat; Eosin-Hãmatoxylin.

Fig. 42. Hämoglobinhaltige Zelle des Knochenmarks mit strahliger Anordnung des Zellleibes; Formolpräparat; Eosin-Hämatoxylin.

Fig. 43. Dasselbe; Trockenpräparat; Eosin-Bämatoxylin.

Fig. 44-48. Hämoglobinhaltige Zellen des Knochenmarks wit rother Granulirung der Kernperipherie, bezw. der Kernstelle; Trockenpräparat; Eosin-Methylenblau.

Fig. 49. Rothes Blutkörperchen aus dem Knochenmark mit centralem Korn. 